\title{
MODELACIÓN, SIMULACIÓN
}

\section{PROCESSES MODELING, SIMULATION AND AUTOMATIZATION IN THE MANAGEMENT OF UNIVERSITY ACADEMIC SERVICES}

Ana Elizabeth Congacha Aushay ${ }^{1}$

Víctor J. García

1. Ingeniera en Sistemas. Magister en Gerencia informática. Diplomado en Diseño Curricular por Competencias. Diplomado en Manejo de la Información a través del Internet. Universidad Nacional de Chimborazo, Docente de la Carrera de Ingeniería en Sistemas y Computación, Riobamba-Ecuador. E-mail: acongacha@unach.edu.ec

2. Ingeniero en Electrónica. MSc en Ingeniería de Control. PhD en Ciencia e Ingeniería de Materiales. Universidad Nacional de Chimborazo, Investigador Prometeo, RiobambaEcuador. E-mail: vgarcia375@gmail.com

\section{Citación sugerida:}

Congacha Aushay, A.E. y García, V.J. (2017). Modelación, simulación y automatización de procesos en la gestión de servicios académicos universitarios. 3C Tecnología: glosas de innovación aplicadas a la pyme, 6(2), 32-51. DOI: <http://dx.doi.org/10.17993/3ctecno.2017.v6n2e22.32-51>. 


\section{RESUMEN}

Comúnmente, en el ámbito universitario la estructura organizativa se caracteriza en que cada departamento se centra en resolver las tareas asignadas. Sin embargo, el servicio entregado es el resultado de la interacción transversal de diferentes áreas verticales de la institución. Esta compleja interacción sumada a la gestión y administración con procedimientos de precaria sistematización que demandan de manera importante la intervención humana, impactan significativamente la eficiencia y eficacia de la gestión de servicios académicos (GSA). Así, nos planteamos como objetivo desarrollar una plataforma informática que de soporte a la GSA como el de las Prácticas Pre-profesionales (PPP) de la Carrera de Sistemas y Computación. Para lograr nuestro objetivo sistematizamos el proceso de selección de la herramienta informática requerida, modelamos el proceso de servicio, validamos el desempeño del modelo y automatizamos el proceso de gestión de las PPP. Nuestra metodología permitió identificar a Bizagi Studio como la tecnología apropiada por su funcionalidad, escalabilidad y capacidad de integración. Además, nuestros resultados muestran que la metodología propuesta permitió desarrollar una plataforma eficiente y eficaz para la gestión de las PPP.

\section{ABSTRACT}

The organizational structure of a university is characterized by each department focusing on solving the tasks assigned. However, the service delivered is the result of the cross-sectional interaction of different vertical areas of the institution. The complex interplay between organizational units and the precarious systematization of the management and administration process - which usually demand a major human intervention - threatens the efficiency and effectiveness of the delivered services. Thus, we aimed to develop a computer platform that supports the MES as the Pre-Professional Practices (PPP) of the Systems and Computing Career. We systematized the selection process of IT tools, modeled the PPP service process, validated the model performance and automated the PPP management process. Our method allowed us to identify Bizagi Studio as the proper technology, because of its functionality, scalability and integration capacity. Also, our results showed the proposed method allows to develop an efficient and effective platform for PPP management.

\section{PALABRAS CLAVE}

Sistemas de administración de procesos de negocio, simulación, BPM, BPMS, BPMN.

\section{KEY WORDS}

Business process management systems, simulation, BPM, BPMS, BPMN. 


\section{INTRODUCCIÓN}

El acelerado proceso de cambio que, a nivel mundial, se ha venido desarrollando en todos los ámbitos del quehacer humano, la globalización, influencia y alienta cada vez más la agilidad de los procesos en las organizaciones.

La gestión de procesos de negocio (BPM), es una disciplina que se convierte en un medio para lograr los objetivos del negocio (Jeston \& Nelis, 2015). En este campo las herramientas de gestión de procesos de negocio (BPMS) brindan el soporte para construir aplicaciones BPM pues manejan el ciclo de vida de los procesos de negocio: modelamiento, ejecución, monitorización y optimización.

Un aspecto crucial es la modelación y simulación del proceso, fases que se pueden apoyar utilizando las herramientas incorporadas en las BPMS. La modelación diseña y diagrama un flujo de proceso que permite entender y analizar los procesos con el fin de proponer mejoras de manera iterativa y la simulación evalúa el desempeño del modelo antes de ejecutarlo con diferentes configuraciones y durante largos períodos de tiempo, para reducir las probabilidades de incumplir los requerimientos de negocio.

Bajo este contexto, la necesidad de mejorar la gestión de los procesos académicos es un reto continuo que deben asumir las instituciones de educación superior con miras a la acreditación y cumplimiento de sus objetivos estratégicos institucionales. En este sentido, el plan estratégico de desarrollo institucional 2012-2016 (PEDI) de la Universidad Nacional de Chimborazo (UNACH), determina como una de sus políticas el mejoramiento e innovación de los procesos académicos, administrativos, de investigación y de vinculación, con sujeción a las disposiciones constitucionales, legales y reglamentarias.

Como consecuencia, la presente investigación tiene como objetivo modelar, simular y automatizar soluciones de mejora de procesos basadas en BPM en el ámbito universitario considerando como muestra al proceso de gestión de PPP de la Carrera de Sistemas y Computación (Congacha, 2015). Así, la siguiente estructura los procesos serán modelados siguiendo la notación internacional BPMN (Business Process Model and Notation), llevándolos desde el modelado, luego a la simulación utilizando el modelador, Bizagi Modeler hasta la automatización, apoyados por una BPMS. 


\section{METODOLOGÍA}

Este estudio se llevó a cabo siguiendo 4 etapas, las cuales son descritas a continuación:

\subsection{SELECCIÓN DE LA SUITE BPM}

Para la selección de la BPMS se utilizaron los resultados de los informes de análisis de tecnologías de las consultoras Forrester (Richardson \& Miers, 2013), Gartner (Sinur \& Hill, 2010) y del instituto de ingeniería de software experimental Fraunhofer (Adam, Riegel, \& Koch, 2013). También se consideraron las recomendaciones generadas por la herramienta de software en línea que proporciona Technology Evaluation Centers (TEC) en términos de las necesidades particulares de la institución, requerimientos específicos de la aplicación que se desea. Se realizó una cross-validación de los diferentes resultados y se encontraron coincidencias que dieron soporte a la selección de la suite BPM más apropiada.

Criterios de consultoras: Forrester consideró 59 criterios de evaluación que se encuentran dentro de las categorías: oferta actual, estrategia y presencia en el mercado, identificando 10 proveedores de software BPM más importantes (Véase Gráfico 1).

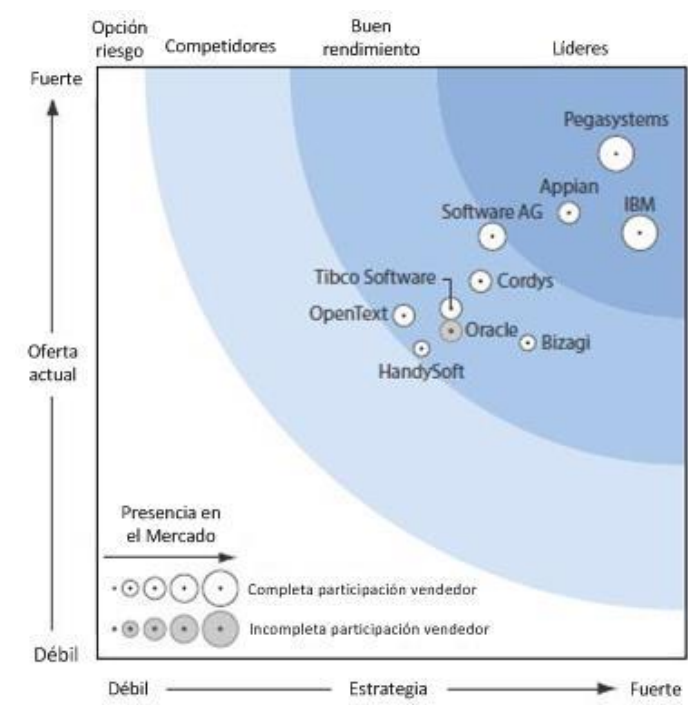

Gráfico 1. Forrester Wave: BPM Suites, Q1 2013. Fuente: Adaptado Forrester Research, Inc.

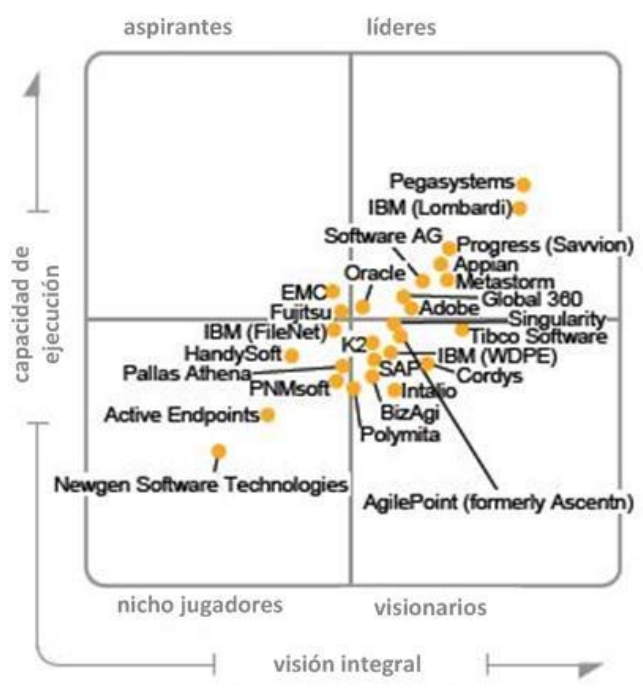

Gráfico 2. Cuadrante Mágico para BPMS. Fuente: Adaptado Gartner (Octubre 2010).

Gartner consideró más de 60 proveedores a nivel mundial y 25 cumplieron los criterios de inclusión (Véase Gráfico 2), fundamentados en escenarios de uso como: soporte a un programa de mejoramiento continuo de procesos, implementación de una solución de procesos específica para una industria o compañía, soporte a una iniciativa de transformación de negocios, soporte al rediseño de una arquitectura orientada a servicios basada en procesos. 
La investigación utilizó el estudio realizado por Gartner en octubre del 2010, debido a que en las posteriores publicaciones se menciona a los iBPMS, una evolución del mercado de BPMS y se centra en un nuevo caso de uso IBO "operaciones de negocio inteligentes", conjuntos de características, aún no consideradas en el presente estudio por factor comparativo.

Criterio instituto de ingeniería de software experimental: En este apartado se analizó la investigación realizada por el instituto alemán Fraunhofer - Ingeniería de Software Experimental (IESE) en colaboración con la empresa de consultoría SP Consulting Ltd. Un total de nueve Suites BPM (Véase Gráfico 3) participaron en el estudio. Cada proveedor presentó su producto a expertos especializados y a científicos, cada participante tuvo que trabajar en los mismos escenarios y sus soluciones fueron comparadas y evaluadas en el contexto del estudio.

Los requisitos se consolidaron en las siguientes categorías: modelado de procesos, proceso de implementación, integración de sistemas, ejecución, gestión en tiempo de ejecución, proceso de control, gobernanza BPM, calidad de corte transversal, administración y configuración.

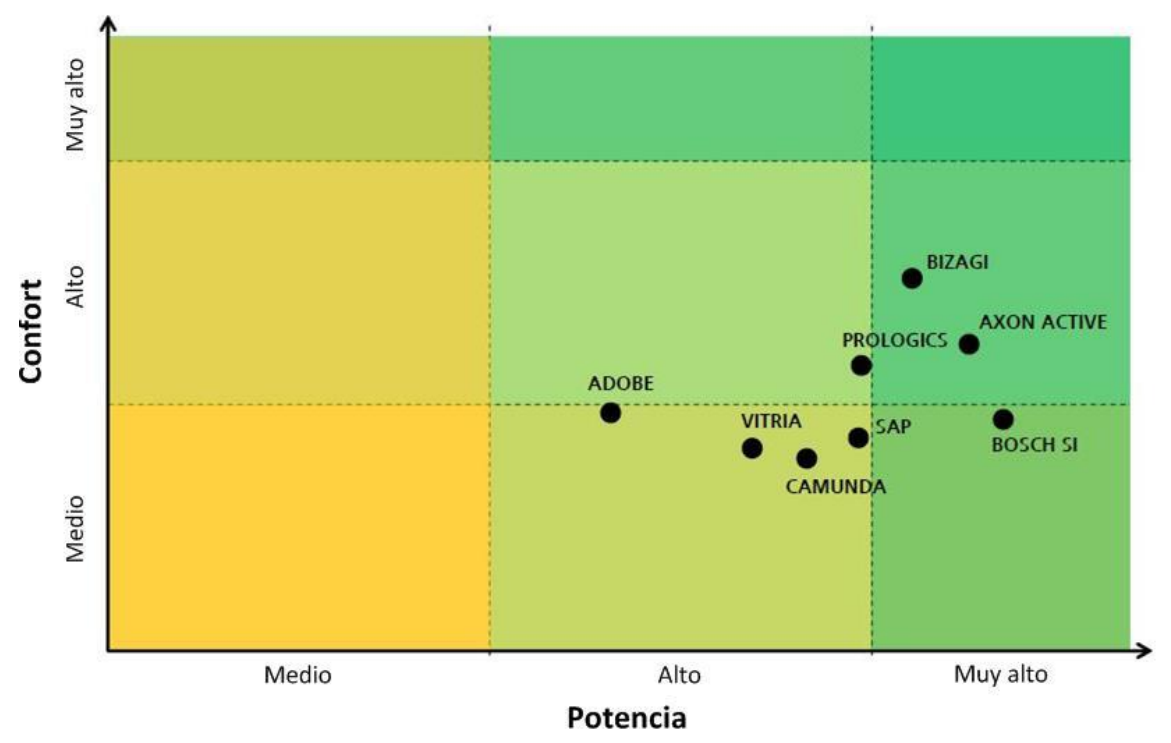

Gráfico 3. Cuadrante de Confort - Potencia de las Suites BPM investigadas. Fuente: Adaptado, Fraunhofer IESE (Diciembre 2013).

Informe de requisitos de alto nivel: En esta sección se ingresó las funcionalidades de la BPMS requeridas por la institución (Véase Tabla 1) en el software en línea que proporciona TEC, generando la lista inicial de proveedores con su producto lo que se constata en el Gráfico 4, determinando las BPMS que cumplen las especificaciones institucionales. 
Tabla 1. Funcionalidades de la BPMS requeridas por la institución.

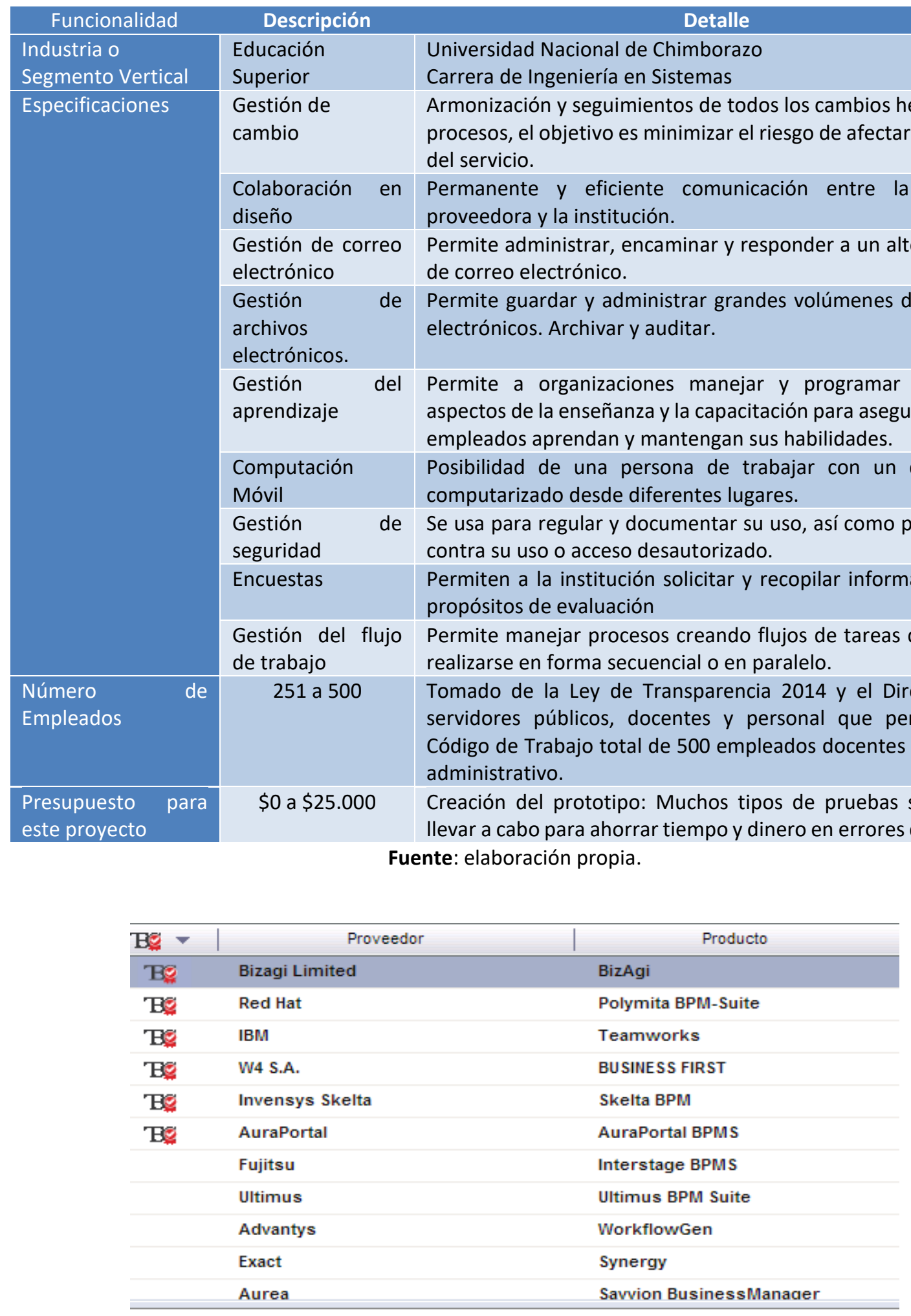

Gráfico 4. Lista inicial para la evaluación de suites BPM.

Fuente: Technology Evaluation Centers. Gestión de los procesos empresariales (BPM). http://itadvisor.technologyevaluation.com 


\subsection{MODELADO DEL PROCESO PPP}

Este trabajo utilizó la metodología BPM:RAD ${ }^{\circledR}$ - Rapid Analysis \& Design (Véase Gráfico 5) para modelar y diseñar el proceso académico, el reglamento de PPP de la Facultad de Ingeniería (UNACHIngeniería, 2013) fue un insumo importante para el desarrollo de esta etapa de la investigación pues permitió especificar los requerimientos de los nodos del proceso, los procesos fueron modelados en Bizagi Modeler siguiendo la notación internacional BPMN.

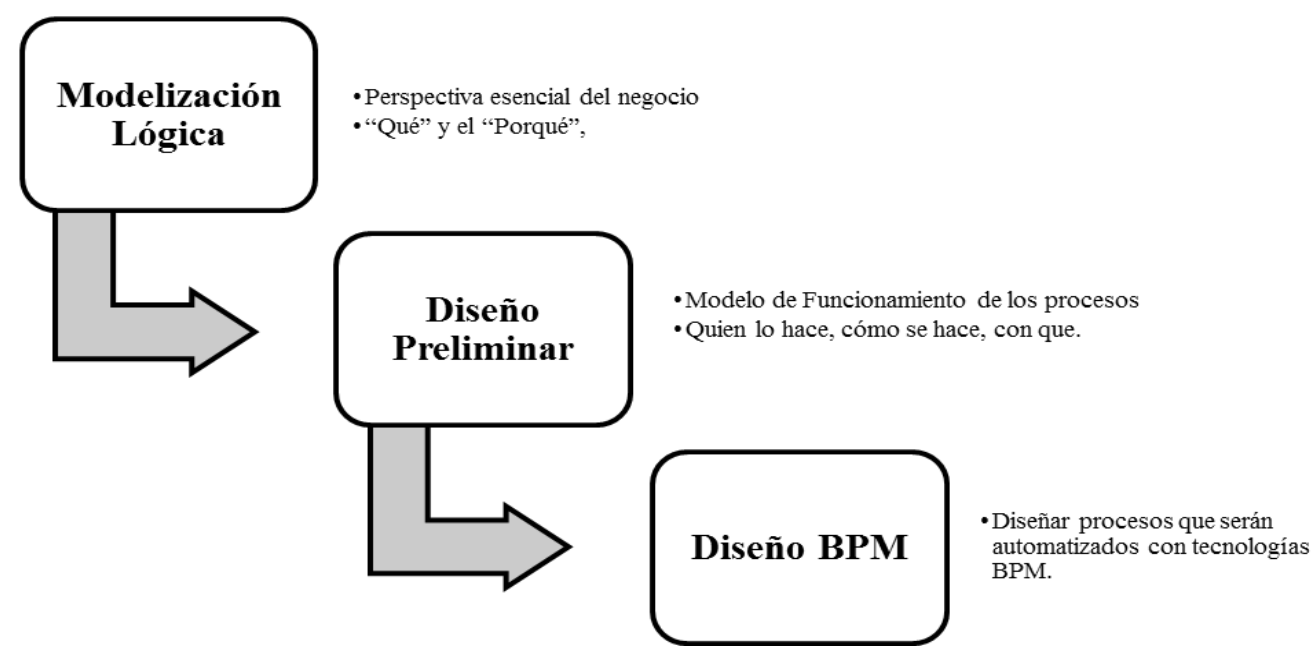

Gráfico 5. Fases metodología BPM: $\mathrm{RAD}^{\circledR}$ - Rapid Analysis \& Design.

Fuente: Elaboración propia 


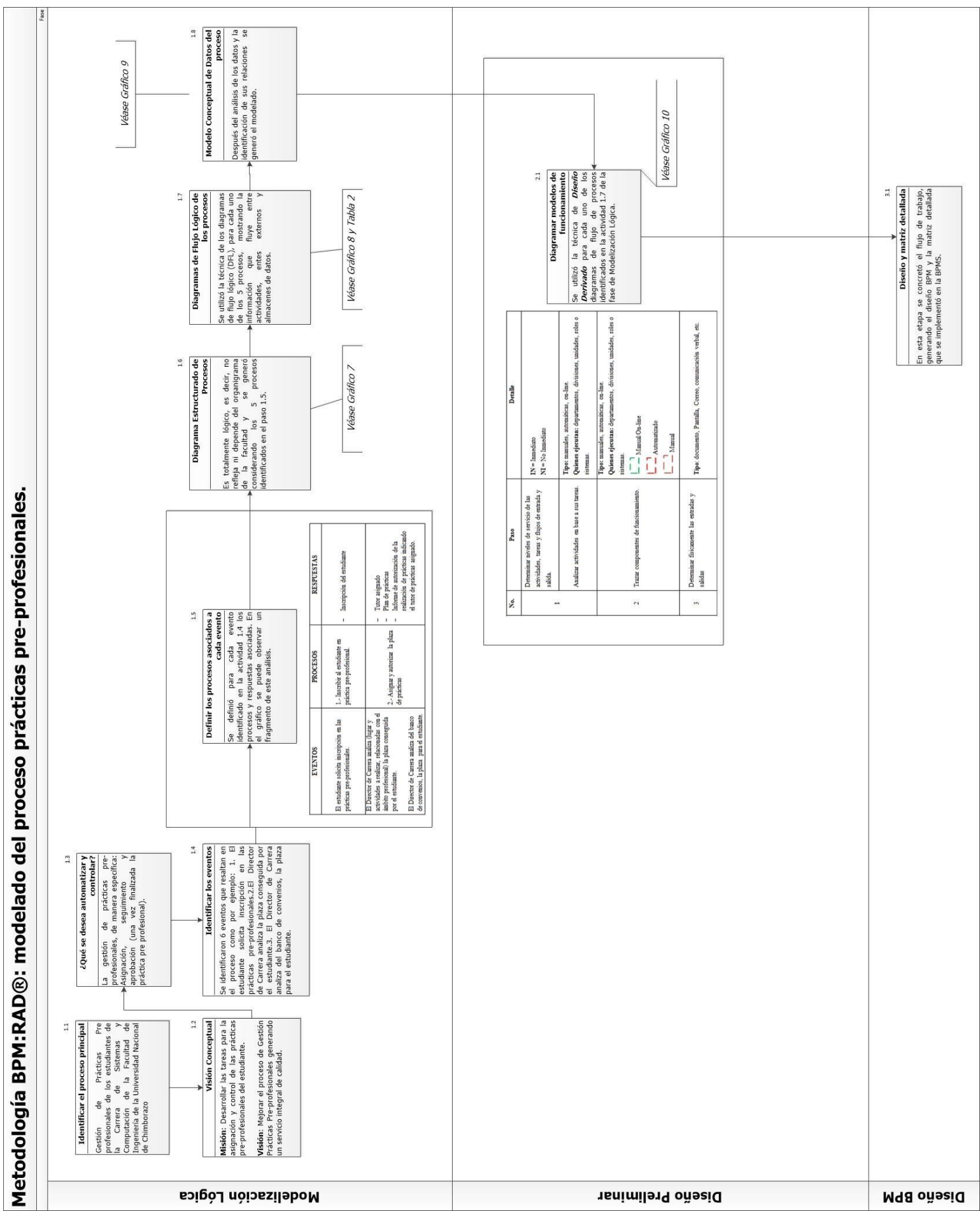

Gráfico 6. Metodología BPM: RAD ${ }^{\circledR}$ aplicada al proceso prácticas pre-profesionales.

Fuente: Elaboración propia. 


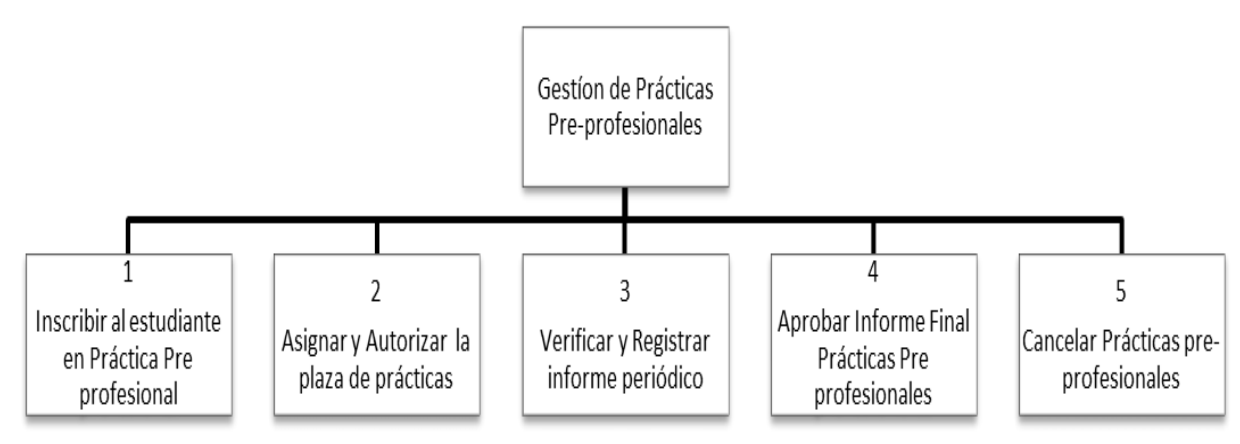

Gráfico 7. Diagrama Estructurado Procesos: Gestión Prácticas

Fuente: elaboración propia.

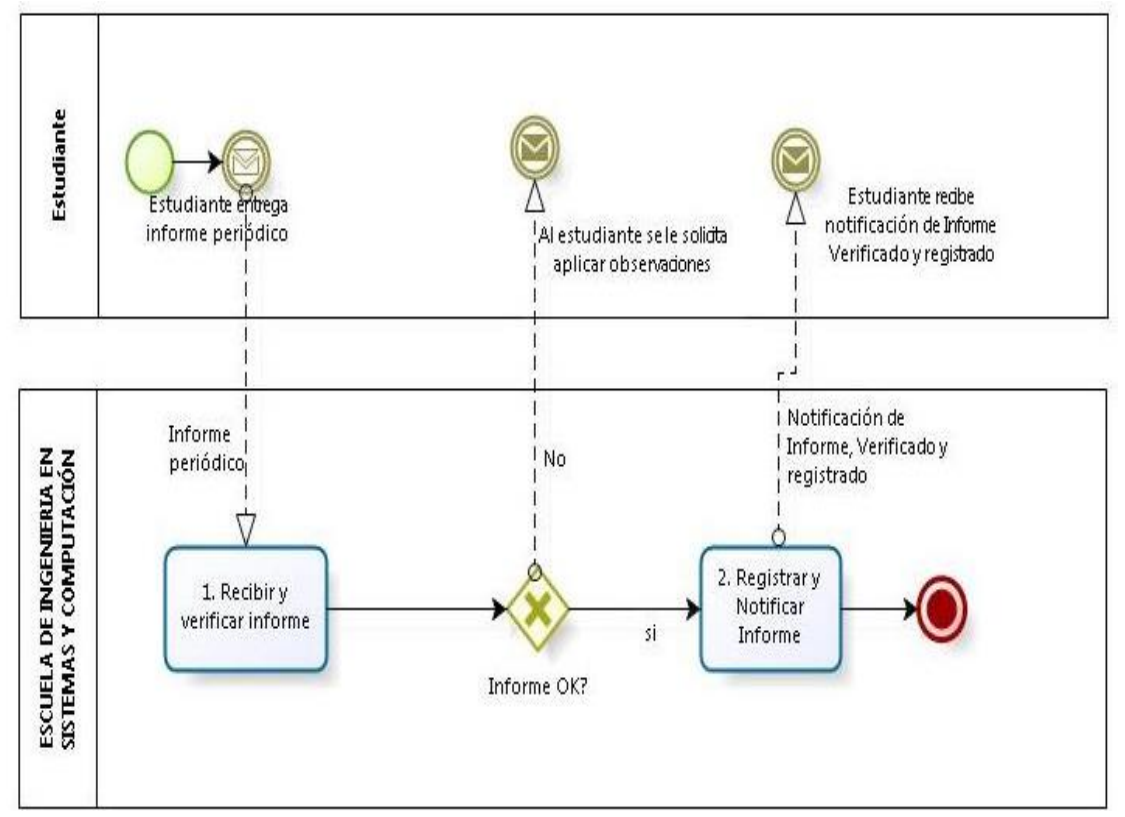

Gráfico 8. Diagrama de Flujo Lógico: Verificar y Registrar informe periódico.

Fuente: elaboración propia.

Tabla 2. Subprocesos y actividades del proceso: verificar y registrar informe.

\begin{tabular}{|c|c|c|}
\hline No. & SubProceso & Actividades \\
\hline 1. & $\begin{array}{l}\text { Recibir y verificar } \\
\text { informe }\end{array}$ & $\begin{array}{l}\text { 1.1 Recibir el informe periódico de actividades realizadas en las prácticas pre } \\
\text { profesionales. } \\
\text { 1.2 Verificar que el informe periódico cumpla lo requerido como: formato, } \\
\text { archivo en donde conste la firma del Jefe inmediato de la plaza. }\end{array}$ \\
\hline 2. & $\begin{array}{l}\text { Registrar y notificar } \\
\text { Informe }\end{array}$ & $\begin{array}{l}\text { 2.1 Registrar o almacenar informe periódico en la Base de Datos. } \\
\text { 2.2 Enviar una notificación que el informe periódico está correcto. }\end{array}$ \\
\hline
\end{tabular}

Fuente: Elaboración propia. 
3C Tecnología (Edición 22) Vol.6 - № 2

Junio -septiembre'17, 32- 51

(3c) tecnología

Área de Innovación y Desarrollo, S.L.

ISSN: $2254-4143$

DOI: http://dx.doi.org/10.17993/3ctecno.2017.v6n2e22.32-51

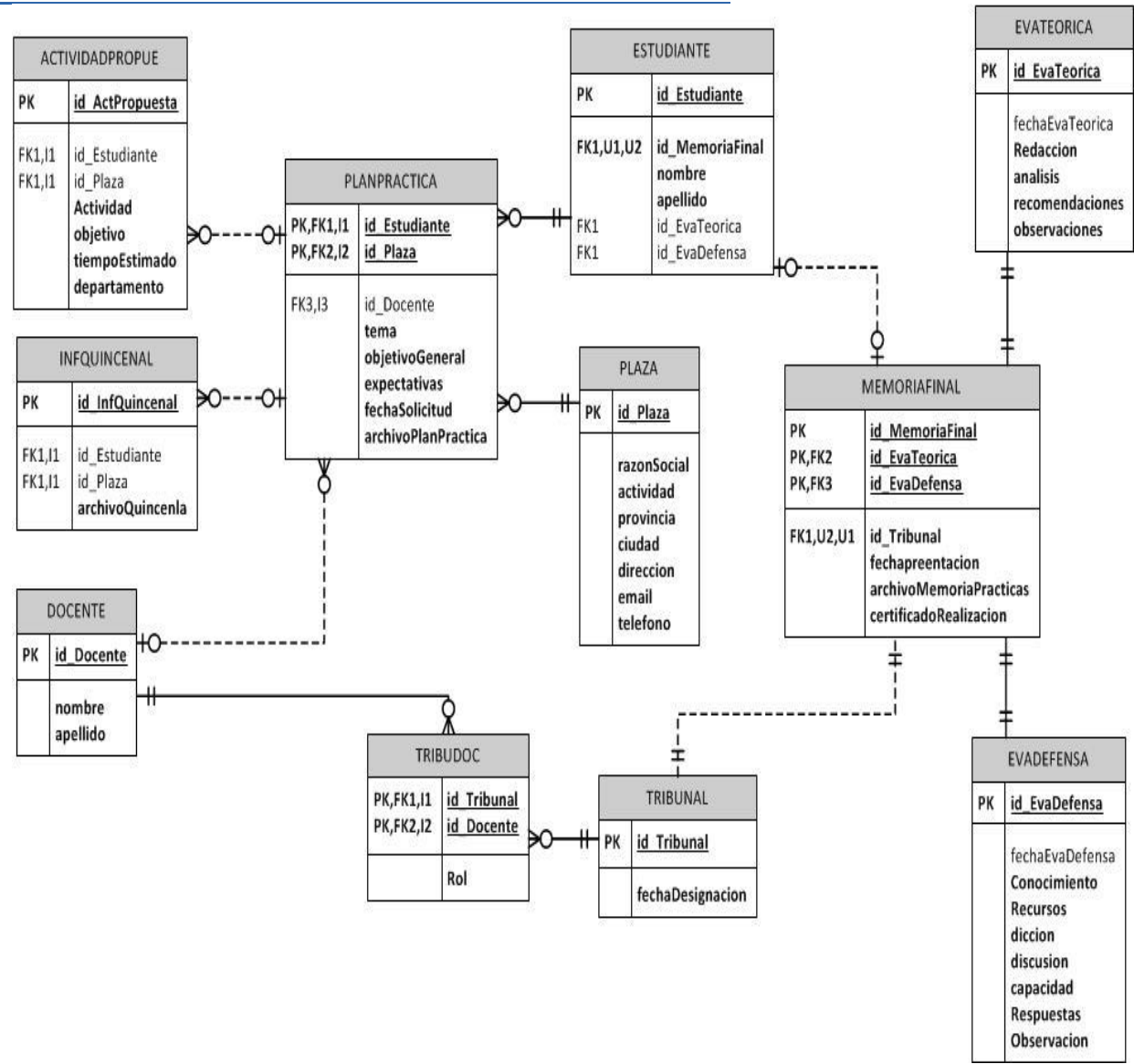

Gráfico 9. Modelo Entidad -Relación: Gestión de Prácticas Pre-profesionales.

Fuente: Elaboración propia.

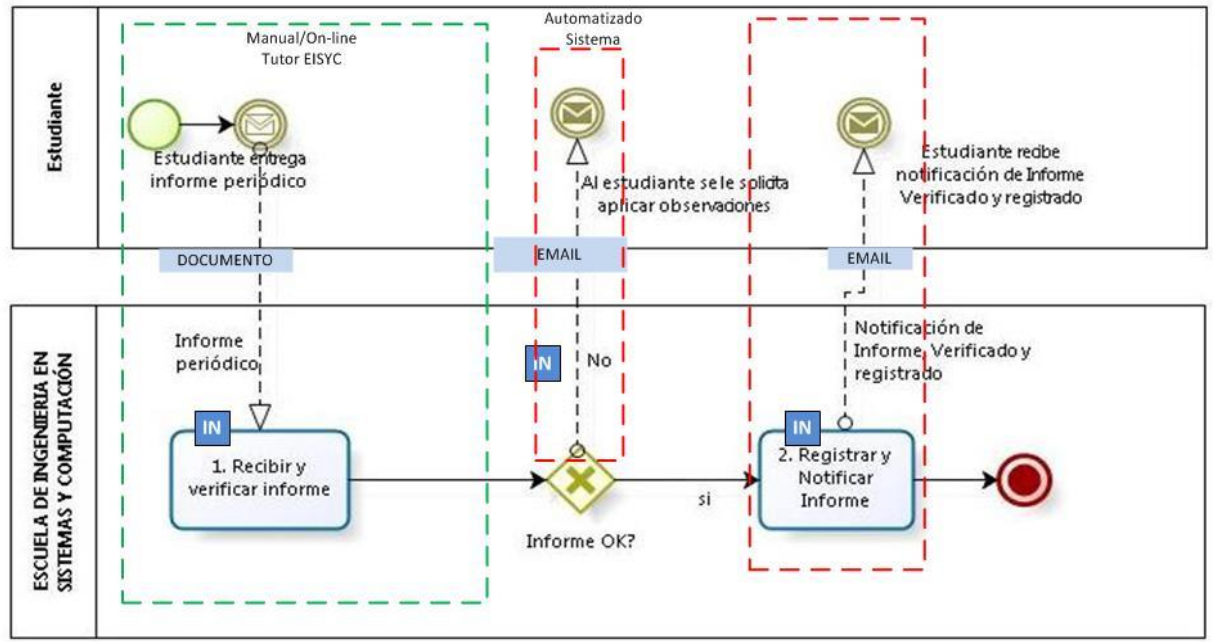

Gráfico 10. Modelo de Funcionamiento: Verificar y Registrar informe quincenal.

Fuente: Elaboración propia. 


\subsection{AUTOMATIZACIÓN DEL MODELO GENERADO EN LA BPMS}

Se generó el modelo de datos a partir del modelo entidad-relación, se procedió a crear las interfaces que permiten interactuar con el flujo de proceso automatizado, se aplicó reglas, estableciendo que procedimientos deben ser ejecutados y las condiciones que deben ser evaluadas y controladas. También se asignaron los usuarios a cada una de las actividades del proceso, esto permitió direccionar adecuadamente el trabajo al recurso pertinente.

\subsection{VALIDACIÓN}

Para evaluar el desempeño de la solución generada, se utilizó el estándar BPSim (Wfmc, Status, Attribution, \& By, 2013) en los escenarios de simulación: validación del proceso y análisis de tiempo, del modelador Bizagi Modeler, en donde la aleatoriedad es simulada usando funciones de distribución de probabilidades de ocurrencia para así establecer los flujos de secuencia y el enrutamiento de "tokens", además para reflejar la variabilidad en los tiempos de proceso y en la ejecución de cada actividad.

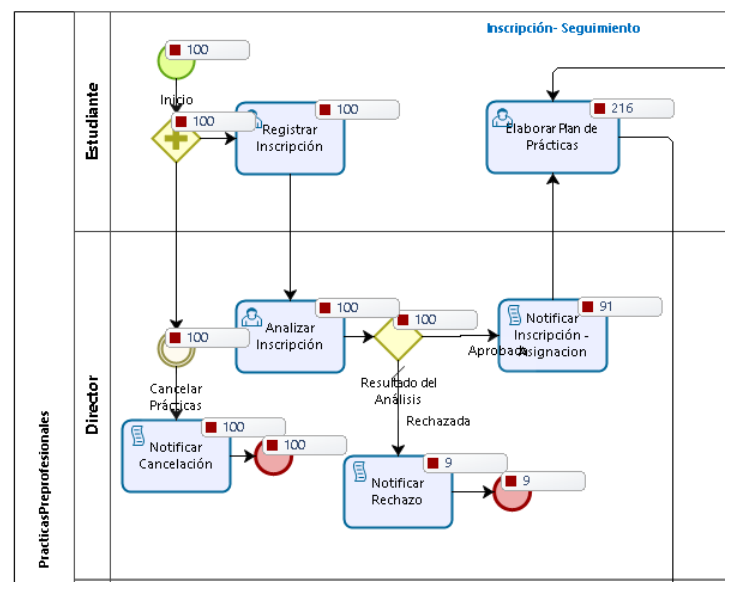

Gráfico 11. Fragmento de la Simulación: Ejecución de la validación del proceso.

Fuente: Elaboración propia.

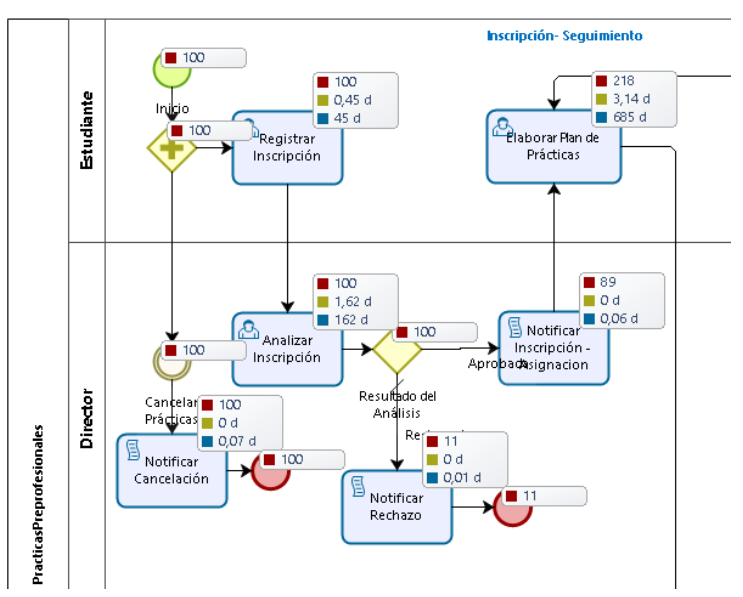

Gráfico 12. Fragmento de la Simulación: Ejecución de análisis de tiempo.

Fuente: Elaboración propia.

En validación de proceso las propiedades del escenario de simulación fueron unidad de tiempo: días, duración: 200 días (Véase Gráfico 11). Se definieron las probabilidades de los caminos de las compuertas a partir de datos históricos, como ejemplo de las probabilidades de los flujos de secuencia podemos mencionar los siguientes: número de llegadas 100 y la compuerta resultados del análisis configurada con valores de Aprobada $90 \%$, Rechazada $10 \%$.

En el análisis de tiempo se definió el intervalo entre la generación de instancias de proceso y los tiempos estimados para cada actividad (véase Gráfico 12). Para la primera se utilizó un valor constante en unidades expresadas en número de días y para la segunda una distribución de probabilidades discreta de Poisson (Johnson, Kemp, \& Kotz, 2005). 
La distribución de Poisson se emplea para describir la demanda (necesidades) de servicios con una variable aleatoria discreta que asume valores enteros $(0,1,2,3,4,5$ y así sucesivamente).

Al asumir esta distribución estamos considerando que se cumplen los siguientes enunciados: a) La probabilidad de que exactamente un estudiante inicie su proceso de PPP es un número muy pequeño y es constante para cada intervalo de tiempo (por ejemplo en un segundo). b) La probabilidad de que dos o más estudiantes lleguen en un intervalo de un segundo es tan reducida que podemos asignarle un valor cero. c) El número de estudiantes que llegan en determinado intervalo de un segundo es independiente del momento en que el intervalo de un segundo ocurre durante la hora de gran afluencia de estudiantes. d) El número de llegadas en cualquier intervalo de un segundo no depende del número de arribos de cualquier otro intervalo de un segundo.

La simulación permitió comprobar número de instancias completadas, tiempo promedio por actividad, tiempo total de procesamiento por entrada.

ÍNDICE DE EFICIENCIA: PARA MEDIR EL ÍNDICE DE EFICIENCIA DEL SISTEMA DE GESTIÓN DE PPP BASADO EN TECNOLOGÍA BPM RESPECTO A LA ADMINISTRACIÓN ACTUAL TOMAMOS LA FÓRMULA:

$$
\text { Eficiencia }=\left|\frac{V_{t}-V_{t-1}}{V_{t-1}}\right| * 100 \%
$$

Donde $V_{t}$ es el valor que representa el número de días que demora el sistema automatizado y $V_{t-1}$ es el valor que representa el número de días que demora el sistema manual. Para definir la media general del sistema se toma en cuenta la media geométrica dada por:

$$
\text { Media Geométrica }=\sqrt[3]{E_{1} * E_{2} * E_{3}}
$$

Donde $E_{1}, E_{2}, E_{3}$ es el índice de eficiencia calculado para las fases de inscripción, seguimiento y aprobación respectivamente, según ecuación (1).

\section{RESULTADOS Y DISCUSIÓN}

A continuación, se presentan los resultados obtenidos para cada una de las etapas enunciadas en materiales y métodos.

\subsection{SELECCIÓN HERRAMIENTA BPMS}

Por medio de la intersección de los resultados generados por las consultoras Gartner, Forrester y el instituto Fraunhofer se logró identificar como elemento común la plataforma Bizagi, en los conjuntos de resultados de partida (Véase Gráfico 13). Además, el informe de Technology Evaluation Centers determinó que Bizagi es la herramienta tecnológica que apoya y cumple con los requisitos de alto nivel. La Gráfico 14 visualiza las debilidades y fortalezas de los productos seleccionados, basadas en los requisitos institucionales requeridos. Los números fuera de la gráfica corresponden a los números: 
1) Educación, 2) Funcionalidades comunes, 3) Número de empleados y 4) Presupuesto. Los números en el medio $(0,20,40,60,80,100)$ representan valores porcentuales que cuantifican la habilidad del producto para satisfacer las necesidades.

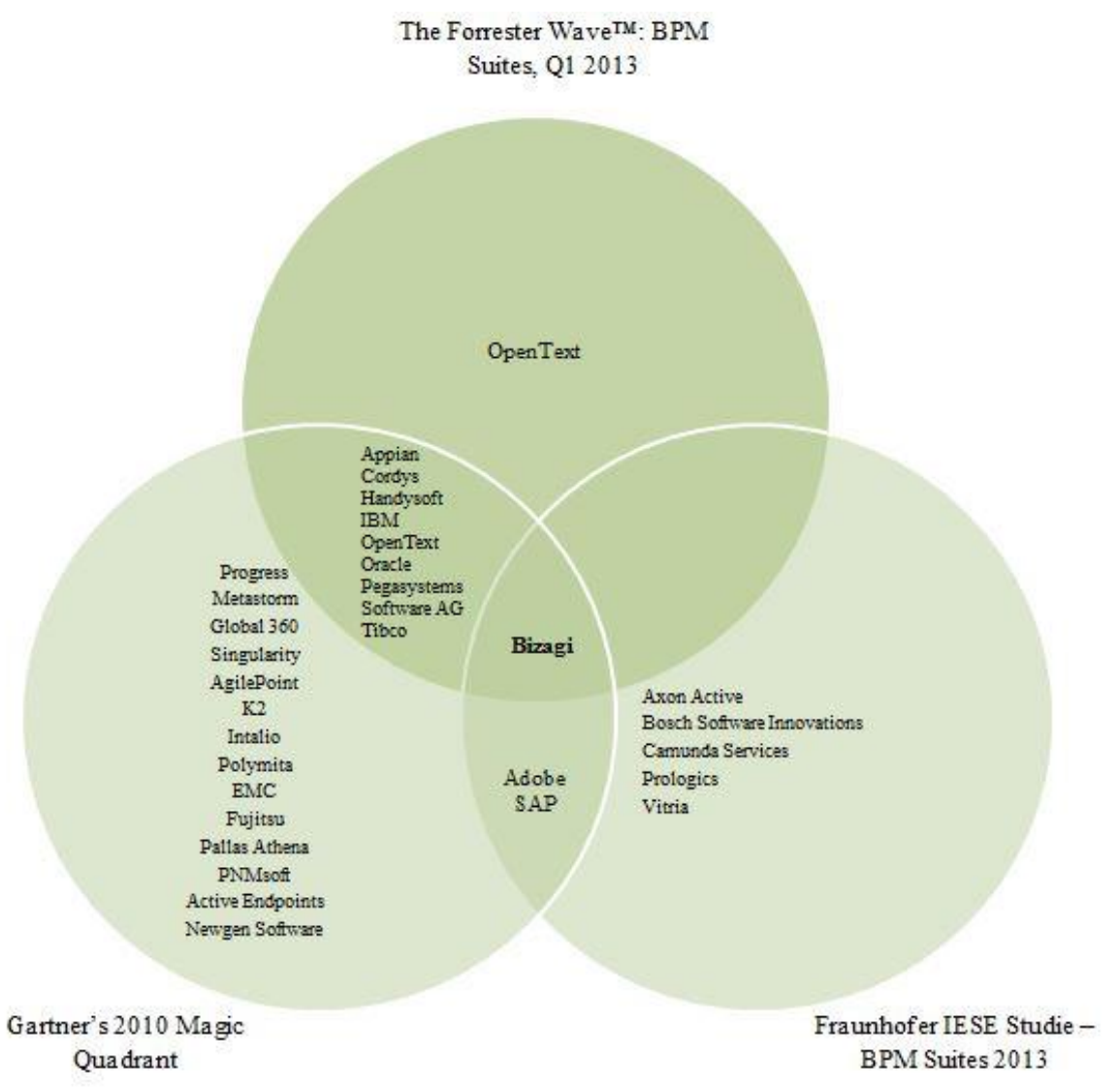

Gráfico 13. Intersección Suites BPM.

Fuente: Elaboración propia.
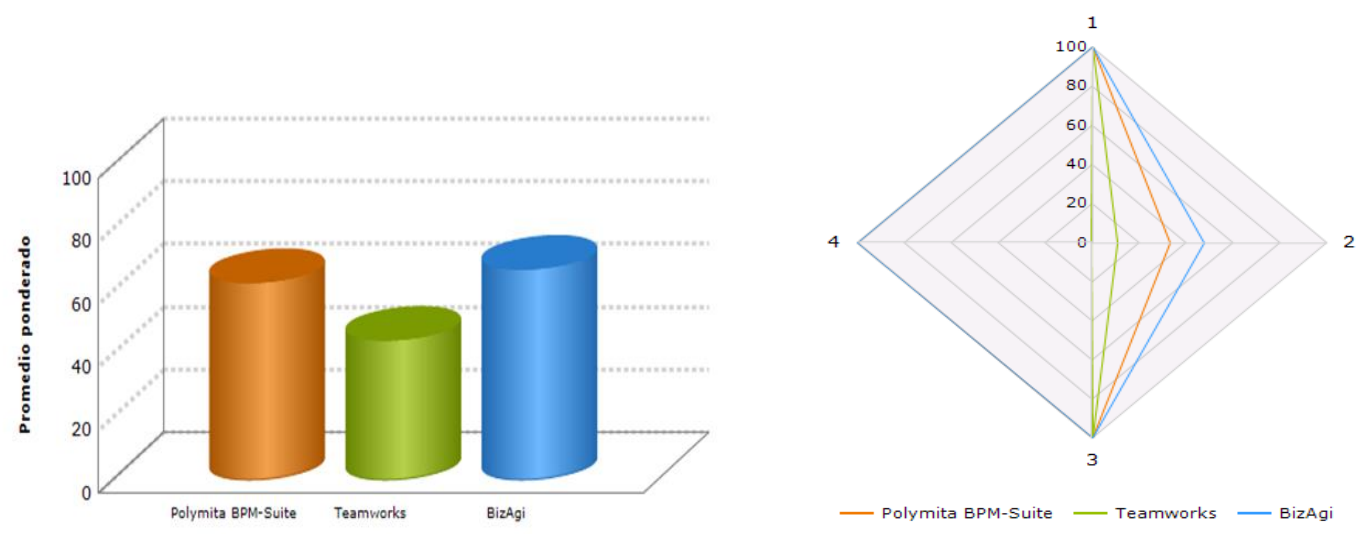

Gráfico 14. Análisis de debilidades y fortalezas basadas en los requisitos.

Fuente: Technology Evaluation Centers. Gestión de los procesos empresariales (BPM).

http://itadvisor.technologyevaluation.com 
Adicionalmente, y para la toma de decisiones, se consideró la existencia de: soporte internacional 24/7, soporte local, casos de éxito, material de apoyo, profesionales con experiencia en el país y características del proveedor como: presencia en el país, oferta de capacitación, compromiso de garantía sobre funcionamiento.

La síntesis de los resultados obtenidos en la selección de la suite BPM identificó a Bizagi como la plataforma de negocios digitales idónea por su funcionalidad, escalabilidad y capacidad de integración apoyando los requerimientos específicos de la UNACH.

\subsection{MODELADO DEL PROCESO DE PPP}

La aplicación de la metodología $B P M: R A D^{\circledR}$ para modelar y diseñar procesos orientados a tecnologías $B P M$, el seguimiento de su enfoque y técnicas fueron factores esenciales para controlar el flujo de trabajo del escenario en cuestión, permitiendo diagramar claramente cada una de las tareas que componen el proceso, definiendo variables de estado, tiempos de espera, usuarios responsables de tareas, tiempos del proceso y por tarea, en consecuencia, se generó el Diseño BPM (Véase Gráfico 15) implementado con la ayuda de la herramienta BPMS, Bizagi Studio.

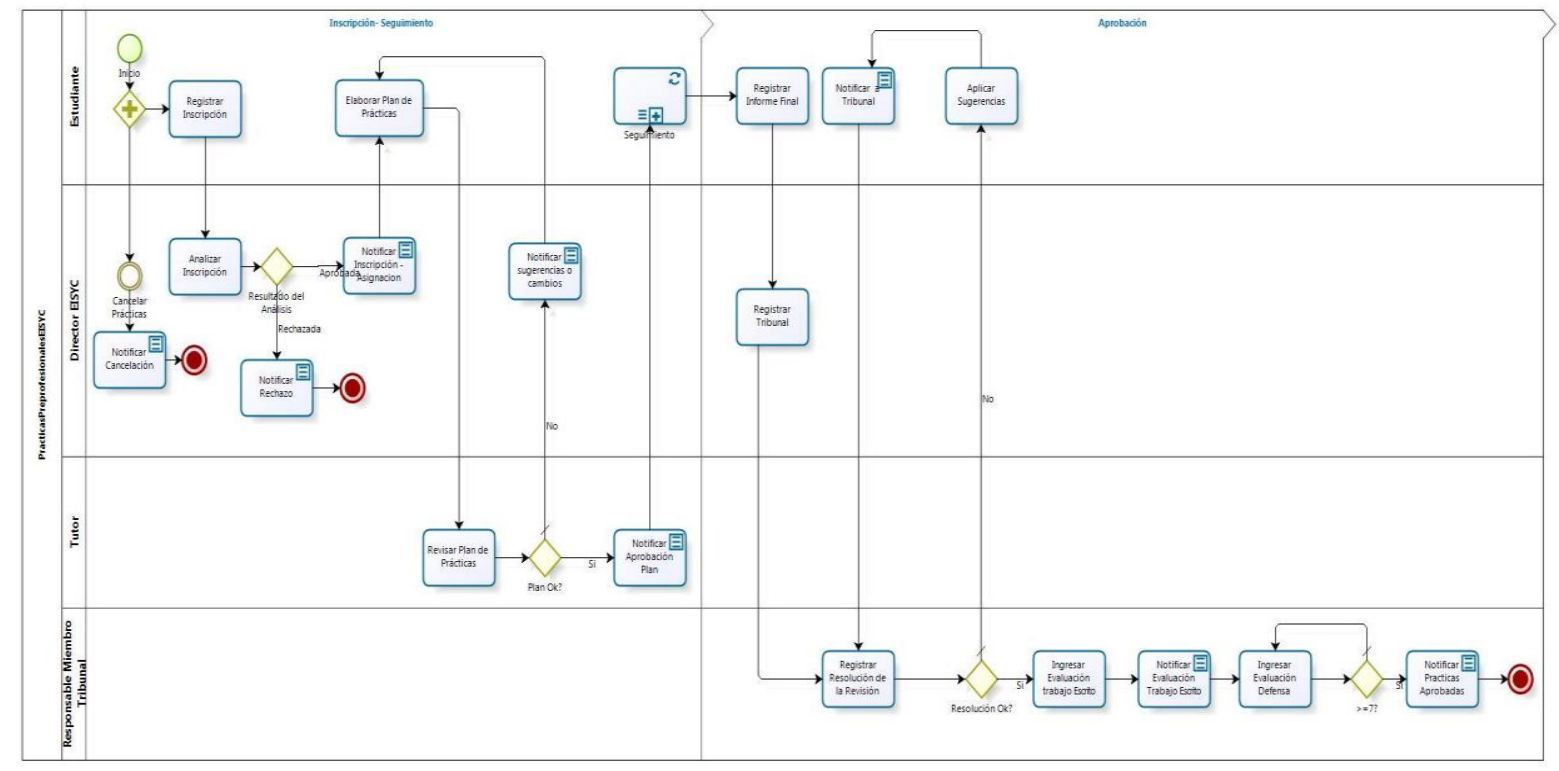

Gráfico 15: Diseño BPM: Gestión de Prácticas Pre-profesionales.

Fuente: Elaboración propia.

\subsection{AUTOMATIZACIÓN DEL MODELO GENERADO EN LA BPMS}

Para transformar el modelo de proceso generado en una aplicación y flujo de trabajo real, se obtuvo el modelo de datos relacional (Véase Gráfico 16) que describe los detalles de cómo se almacenan los datos en el ordenador utilizando la herramienta BPMS. Se crearon las formas o interfaces de usuario que permiten la interacción con la aplicación a través de un portal web de trabajo, cada actividad 
tiene una forma asociada que muestra información del proceso. A modo de ejemplo, en el Gráfico 17 se visualiza la forma: Registrar inscripción.

Además, se establecieron las reglas de negocio que garantizan una correcta ejecución de los procedimientos y las condiciones que deben ser evaluadas y controladas en el flujo de proceso. En la Tabla 3, se puede observar ejemplos de compuertas divergentes implementadas en el sistema y se definió participantes para cada actividad del proceso, asignando criterios y reglas de asignación.

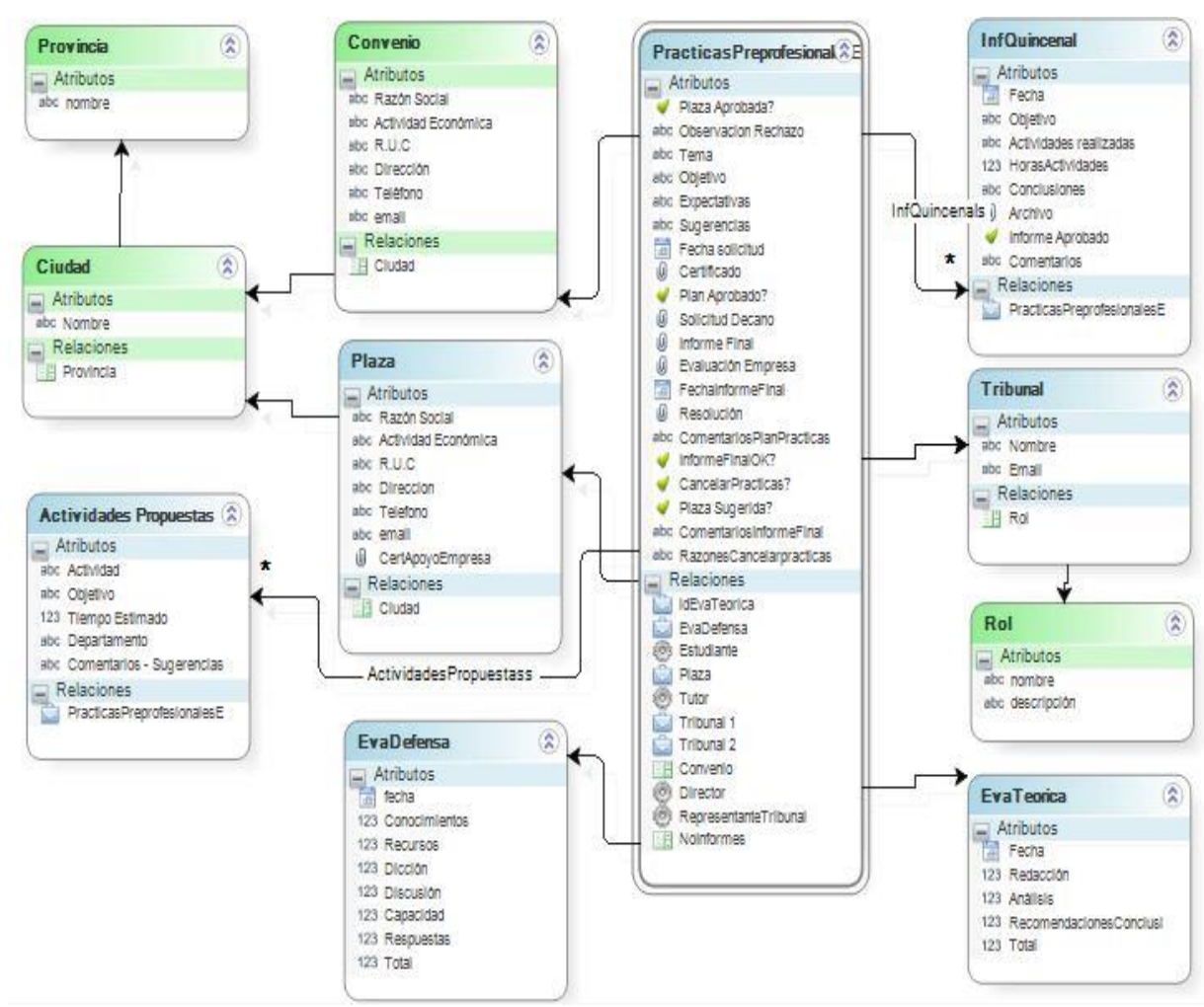

Gráfico 16: Modelo de Datos: Gestión de Prácticas Pre-profesionales.

Fuente: Elaboración propia. 


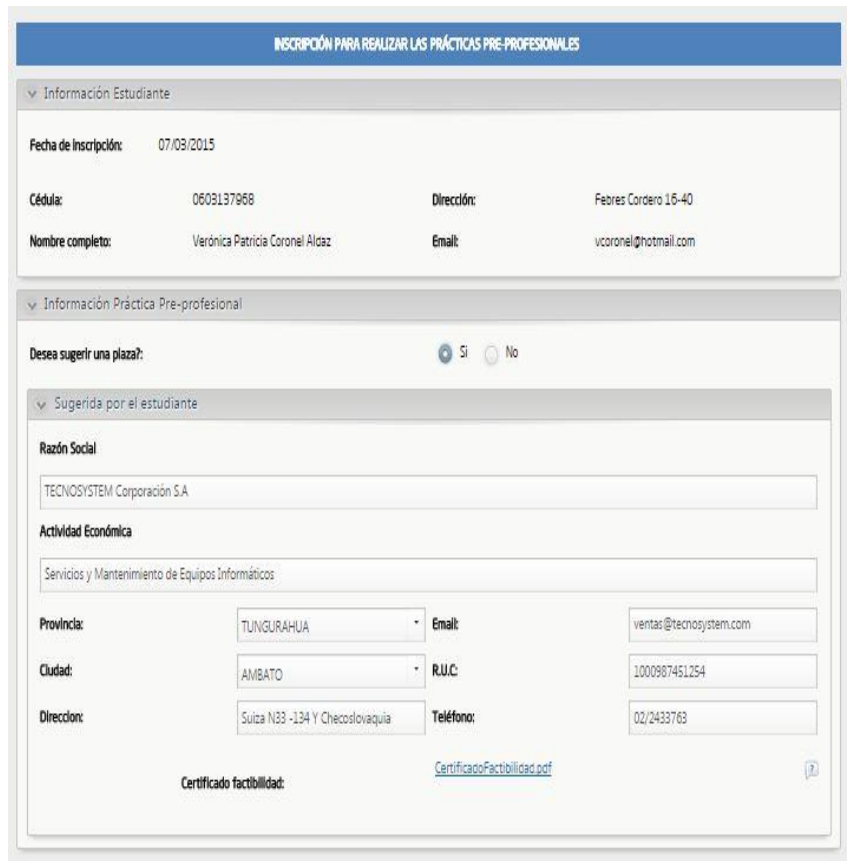

Gráfico 17. Formulario Registrar inscripción.

Fuente: Elaboración propia.

Tabla 3. Ejemplos de rutas utilizadas en la automatización del Proceso PPP.

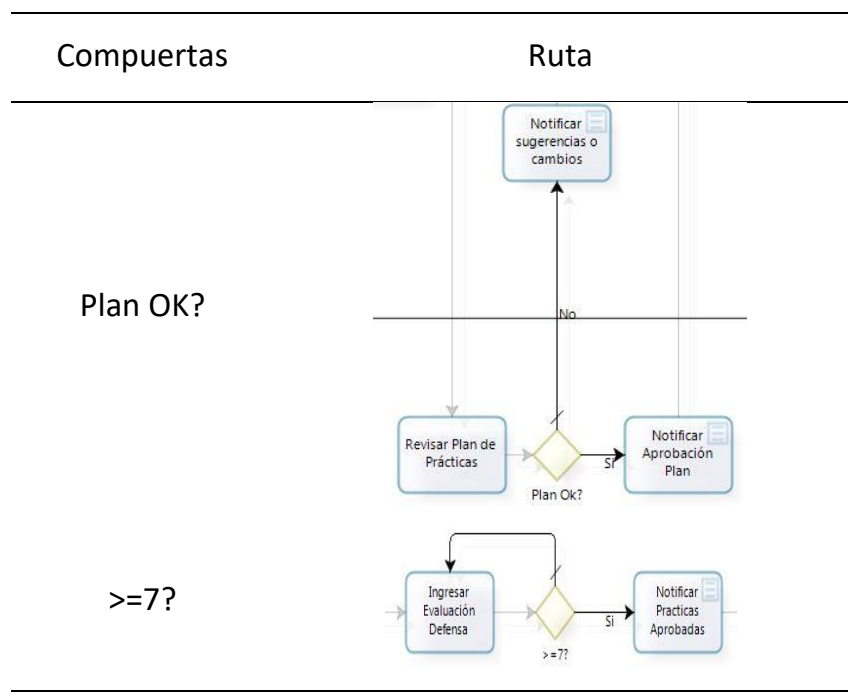

Fuente: Elaboración propia. 


\subsection{VALIDACIÓN}

En la Tabla 4 se presentan los resultados generados al ejecutar la simulación que permitió la validación del proceso. En la tabla 4 se puede observar que el funcionamiento es el esperado dado que el número de instancias creadas (100) es igual al número de instancias completadas $(91+9)$. Las instancias se encaminan a las diferentes estancias de acuerdo a las probabilidades definidas.

Tabla 4. Resultados de la simulación, validación del proceso. Bizagi.

\begin{tabular}{|c|c|c|}
\hline Nombre & Tipo & $\begin{array}{c}\text { Instancias } \\
\text { completadas }\end{array}$ \\
\hline PracticasPreprofesionales & Proceso & 100 \\
\hline Plan Ok? & Compuerta & 216 \\
\hline Revisar Plan de Prácticas & Tarea & 216 \\
\hline Inicio & Evento de inicio & 100 \\
\hline Elaborar Plan de Prácticas & Tarea & 216 \\
\hline Resultado del Análisis & Compuerta & 100 \\
\hline Notificar Rechazo & Tarea & 9 \\
\hline Notificar Inscripción - Asignacion & Tarea & 91 \\
\hline Notificar sugerencias o cambios & Tarea & 125 \\
\hline Registrar Tribunal & Tarea & 91 \\
\hline Registrar Informe Final & Tarea & 91 \\
\hline Registrar Resolución de la Revisión & Tarea & 93 \\
\hline Ingresar Evaluación trabajo Escrito & Tarea & 91 \\
\hline Resolución Ok? & Compuerta & 93 \\
\hline Ingresar Evaluación Defensa & Tarea & 96 \\
\hline Cancelar Prácticas & Evento intermedio & 100 \\
\hline Notificar Aprobación Plan & Tarea & 91 \\
\hline Notificar a Tribunal & Tarea & 2 \\
\hline Notificar Evaluación Trabajo Escrito & Tarea & 91 \\
\hline Aplicar Sugerencias & Tarea & 2 \\
\hline$>=7 ?$ & Compuerta & 96 \\
\hline Notificar Practicas Aprobadas & Tarea & 91 \\
\hline Registrar Inscripción & Tarea & 100 \\
\hline Analizar Inscripción & Tarea & 100 \\
\hline Seguimiento & Tarea & 91 \\
\hline Notificar Cancelación & Tarea & 100 \\
\hline
\end{tabular}

Fuente: Elaboración propia.

En la Tabla 5 se muestran los resultados del análisis de tiempo. Los resultados en este nivel proporcionan una idea general del tiempo total para el proceso. Para este caso concreto se define el tiempo que un estudiante demora en realizar sus PPP completando las fases de inscripción, 
seguimiento y aprobación es decir desde el momento en que solicita la inscripción, hasta que su proceso completo de gestión de PPP es aprobado.

Con base en los resultados de esta simulación por ejemplo, en promedio un estudiante puede demorar 106 días, 4 horas, 9 minutos y 48 segundos en completar las fases (inscripción, seguimiento y aprobación) del proceso PPP.

Tabla 5. Resultados de la simulación, análisis de tiempo. Adaptado Bizagi.

\begin{tabular}{|c|c|c|c|c|c|c|}
\hline & & \multicolumn{2}{|c|}{ Instancias } & \multicolumn{3}{|c|}{ Tiempo } \\
\hline Nombre & Tipo & Completadas & Iniciadas & $\begin{array}{l}\text { Máximo } \\
\text { (d) }\end{array}$ & $\begin{array}{l}\text { Promedio } \\
\text { (d) }\end{array}$ & Total (d) \\
\hline PracticasPreprofesionales & Proceso & 100 & 100 & $\begin{array}{l}185 d \\
13 m\end{array}$ & $\begin{array}{l}106 d 4 h \\
9 m 48 s\end{array}$ & $\begin{array}{l}10617 \mathrm{~d} \\
10 \mathrm{~h} 1 \mathrm{~m}\end{array}$ \\
\hline Plan Ok? & Compuerta & 218 & 218 & & & \\
\hline Revisar Plan de Prácticas & Tarea & 218 & 218 & $14 d$ & $\begin{array}{l}4 d 18 h \\
3 m 18 s\end{array}$ & $1036 d$ \\
\hline Elaborar Plan de Prácticas & Tarea & 218 & 218 & $10 d$ & $\begin{array}{c}3 d 3 h \\
24 m 46 s\end{array}$ & $685 d$ \\
\hline Resultado del Análisis & Compuerta & 100 & 100 & & & \\
\hline Notificar Rechazo & Tarea & 11 & 11 & $1 \mathrm{~m}$ & $1 \mathrm{~m}$ & $11 \mathrm{~m}$ \\
\hline $\begin{array}{l}\text { Notificar Inscripción - } \\
\text { Asignacion }\end{array}$ & Tarea & 89 & 89 & $1 \mathrm{~m}$ & $1 \mathrm{~m}$ & $1 \mathrm{~h} 29 \mathrm{~m}$ \\
\hline $\begin{array}{l}\text { Notificar sugerencias o } \\
\text { cambios }\end{array}$ & Tarea & 129 & 129 & $1 \mathrm{~m}$ & $1 \mathrm{~m}$ & $2 \mathrm{~h} 9 \mathrm{~m}$ \\
\hline Registrar Tribunal & Tarea & 89 & 89 & $12 d$ & $\begin{array}{c}5 d 1 h \\
20 m 53 s\end{array}$ & $450 d$ \\
\hline Registrar Informe Final & Tarea & 89 & 89 & $10 d$ & $\begin{array}{c}4 d 18 h \\
36 \mathrm{~m} \mathrm{24s}\end{array}$ & $425 d$ \\
\hline $\begin{array}{l}\text { Registrar Resolución de la } \\
\text { Revisión }\end{array}$ & Tarea & 94 & 94 & $11 d$ & $\begin{array}{c}5 d 7 h \\
39 m 34 s\end{array}$ & $500 d$ \\
\hline $\begin{array}{l}\text { Ingresar Evaluación } \\
\text { trabajo Escrito }\end{array}$ & Tarea & 89 & 89 & $5 d$ & $\begin{array}{c}2 \mathrm{~d} 32 \mathrm{~m} \\
21 \mathrm{~s}\end{array}$ & $180 d$ \\
\hline Resolución Ok? & Compuerta & 94 & 94 & & & \\
\hline $\begin{array}{l}\text { Ingresar Evaluación } \\
\text { Defensa }\end{array}$ & Tarea & 92 & 92 & $17 d$ & $\begin{array}{l}9 \mathrm{~d} 18 \mathrm{~h} \\
31 \mathrm{~m} \mathrm{18s}\end{array}$ & $899 d$ \\
\hline Cancelar Prácticas & $\begin{array}{l}\text { Evento } \\
\text { intermedio }\end{array}$ & 100 & 100 & & & \\
\hline Notificar Aprobación Plan & Tarea & 89 & 89 & $1 \mathrm{~m}$ & $1 \mathrm{~m}$ & $1 \mathrm{~h} 29 \mathrm{~m}$ \\
\hline Notificar a Tribunal & Tarea & 5 & 5 & $1 \mathrm{~m}$ & $1 \mathrm{~m}$ & $5 m$ \\
\hline $\begin{array}{l}\text { Notificar Evaluación } \\
\text { Trabajo Escrito } \\
\end{array}$ & Tarea & 89 & 89 & $1 \mathrm{~m}$ & $1 \mathrm{~m}$ & $1 \mathrm{~h} 29 \mathrm{~m}$ \\
\hline Aplicar Sugerencias & Tarea & 5 & 5 & $2 d$ & $1 d$ & $5 d$ \\
\hline$>=7 ?$ & Compuerta & 92 & 92 & & & \\
\hline $\begin{array}{l}\text { Notificar Practicas } \\
\text { Aprobadas }\end{array}$ & Tarea & 89 & 89 & $1 \mathrm{~m}$ & $1 \mathrm{~m}$ & $1 \mathrm{~h} 29 \mathrm{~m}$ \\
\hline Registrar Inscripción & Tarea & 100 & 100 & $2 d$ & $10 \mathrm{~h} 48 \mathrm{~m}$ & $45 d$ \\
\hline Analizar Inscripción & Tarea & 100 & 100 & $5 d$ & $\begin{array}{l}1 d 14 h \\
52 \mathrm{~m} \mathrm{48s}\end{array}$ & $162 d$ \\
\hline Seguimiento & Tarea & 89 & 89 & $70 d$ & 70 & $6230 d$ \\
\hline Notificar Cancelación & Tarea & 100 & 100 & $1 \mathrm{~m}$ & $1 \mathrm{~m}$ & $1 \mathrm{~h} 40 \mathrm{~m}$ \\
\hline
\end{tabular}

Fuente: Elaboración propia. 
Finalmente, se calculó el índice de eficiencia del proceso PPP (Véase Tabla 6). Así, la fase de inscripción automatizada presentó un $89 \%$ de eficiencia respecto al proceso manual, la fase de seguimiento un $20 \%$ y la fase de aprobación un $91 \%$. La eficiencia del proceso automatizado completo se mide por la media de los índices de las fases y está dada por $55 \%$.

Tabla 6. Índice de Eficiencia proceso PPP.

\begin{tabular}{l|c|c|c|}
\hline \multicolumn{1}{|c}{ Fases } & $\begin{array}{c}\text { Manual } \\
\text { (días) }\end{array}$ & $\begin{array}{c}\text { Automatizado } \\
\text { (días) }\end{array}$ & Índice de eficiencia \\
\hline INSCRIPCIÓN & $92,0^{*}$ & $10^{* *}$ & $89 \%$ \\
\hline SEGUIMIENTO & $88,0^{*}$ & $70 * *$ & $20 \%$ \\
\hline APROBACIÓN & $295,0^{*}$ & $26 * *$ & $91 \%$ \\
\hline *tiempo obtenido al calcular la media de la muestra ** tiempo promedio \\
generado en la simulación.
\end{tabular}

Fuente: Elaboración propia.

\section{CONCLUSIONES}

La gestión en una institución universitaria hace que el servicio entregado al cliente sea el resultado de la interacción transversal de diferentes áreas verticales de la estructura organizacional. En este contexto complejo y de mucha incertidumbre, el aporte de nuestro trabajo se orienta a la sistematización, desarrollo y consolidación de los procesos orientados a tecnologías BPM en instituciones universitarias.

Un aspecto relevante en el desarrollo de nuestro trabajo ha sido el abstraer las cualidades esenciales del proceso de gestión, sintetizar, modelar y controlar la dinámica del flujo de trabajo de PPP a través del uso de la BPMS como apoyo a la gestión de procesos de negocio. Así, se ha mostrado que es posible mejorar sustancialmente el grado de cumplimiento de objetivos funcionales en la gestión de procesos académicos.

Como futura extensión de la investigación, dada la creciente demanda de inteligencia operativa se plantea la incorporación de métodos de la ciencia de datos, así como el análisis de la evolución de las herramientas BPMS llamadas iBPMS para dar soporte e inteligencia a los procesos de gestión académica en instituciones universitarias.

\section{AGRADECIMIENTOS}

Se expresa agradecimiento al Proyecto Prometeo de la Secretaria Superior, Ciencia, Tecnología e Innovación de la República del Ecuador. 


\section{REFERENCIAS BIBLIOGRÁFICAS}

Adam, S., Riegel, N., \& Koch, M. (2013). Studie - BPM Suites 2013.

Congacha, A. E. (2015). Los sistemas de gestión de procesos en la administración de prácticas preprofesionales en la Escuela de Ingeniería en Sistemas y Computación de la Universidad Nacional de Chimborazo en el período 2013. Pontificia Universidad Católica del Ecuador. Retrieved from http://repositorio.pucesa.edu.ec/handle/123456789/1211.

Jeston, J., \& Nelis, J. (2015). Business Process Management Practical Guidelines to Successful Implementations. In CEUR Workshop Proceedings (Vol. 1542, pp. 33-36). https://doi.org/10.1017/СВO9781107415324.004.

Johnson, N. L., Kemp, A. W., \& Kotz, S. (2005). Univariate Discrete Distributions (3rd ed.). John Wiley \& Sons.

Richardson, C., \& Miers, D. (2013). The Forrester Wave ${ }^{\mathrm{TM}}$ : BPM Suites, Q1 2013. Forrester Research, Inc., 1-21.

Sinur, J., \& Hill, J. B. (2010). Magic Quadrant for Business Process Management Suites. Reproduction, 164485(October), 1-21. https://doi.org/10.1038/nature00899.

UNACH-Ingeniería. (2013). Reglamento. Riobamba: No. 405-HCD-15-07-2013.

Wfmc, D. N., Status, D., Attribution, C. C., \& By, C. C. (2013). Business Process Simulation Specification, 1-35. Retrieved from http://www.bpsim.org/ 